\title{
Improved ATR Infrared Microanalysis of Thin Multilayered Samples and Included Small Particles
}

\author{
${\text { Thomas J Tague } \mathrm{Jr}^{1} \text { and Gene Hall }}^{2}$ \\ 1 - Bruker Optics, Inc., Billerica, MA 01821 \\ 2 - Department of Chemistry, Rutgers University, 83 Somerset St, New Brunswick NJ, \\ 08901
}

A new reflecting objective for attenuated total reflection (ATR) infrared microanalysis has been designed to provide excellent sample viewing, high infrared sensitivity, and increased ease-of-use. The ATR objective incorporates a unique vertical sliding mechanism, which allows the internal reflection element (IRE) to be positioned out of the field of view (FOV) for viewing of the sample and in the FOV for high throughput infrared analysis, as shown in Figure 1. When the IRE is out of the FOV, contrast enhancement methods can be readily employed, such as visible polarization, fluorescence illumination, darkfield illumination, etc. The IRE is an anvil design with an 80-micron tip providing access to small areas within the sample of interest.

There are five contact pressure levels to choose from, where the contact pressure can be optimized for the sample hardness and tractability. Optimal pressure is indicated by an LED indicator and an audible beep. The IRE is spring loaded, where overpressure would simply move the crystal further up on the slide and no damage can occur. It is desirable to use IRE's with a high index of refraction for three reasons. First, the refractive index of the IRE must be greater than the sample to generate an internal reflection. For carbon filled materials, Ge is the only readily available material with a high enough refractive index to generate a total internal reflection. Second, the high refractive index of Ge (4.0) allows for a $4 x$ magnification of the IR field aperture. This increases the spatial resolution to better than the wavelength of light.

As shown in Figure 2, a multilayer paint chip from an ancient work of art was visualized utilizing crossed polarized light illumination. The IR aperture was visually adjusted to mask each area of interest followed by infrared ATR data collection. Pure spectra were obtained for each component of the chip without contamination from adjacent areas. Little to no diffraction was evident in the resulting spectra.

In order to evaluate the spatial limits of this type of ATR analysis, the yellow particle (6x6 microns in size) was analyzed with the aperture closed to $12 \times 12$ and $24 \times 24$ microns in separate measurements. This projected to analysis areas of $3 \times 3$ and $6 \times 6$ microns, respectively. Some diffraction was evident in the $3 \times 3$ result and was absent in the $24 \times 24$ micron result. 


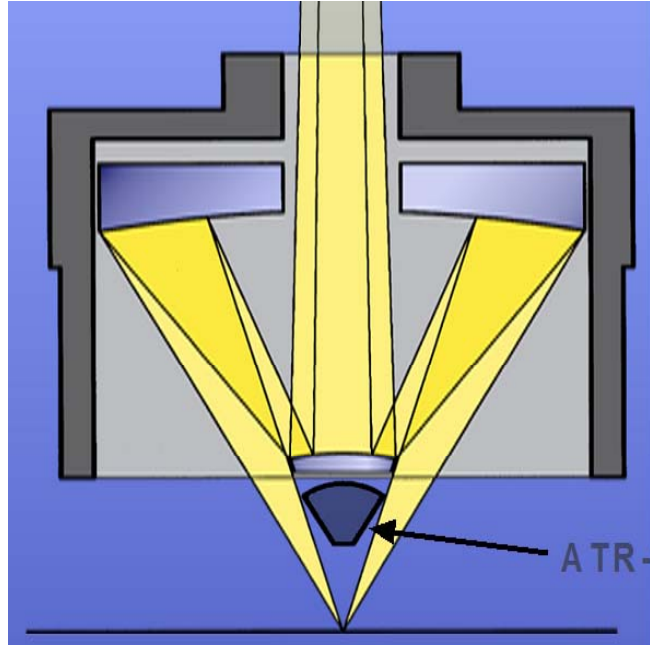

Observation mode

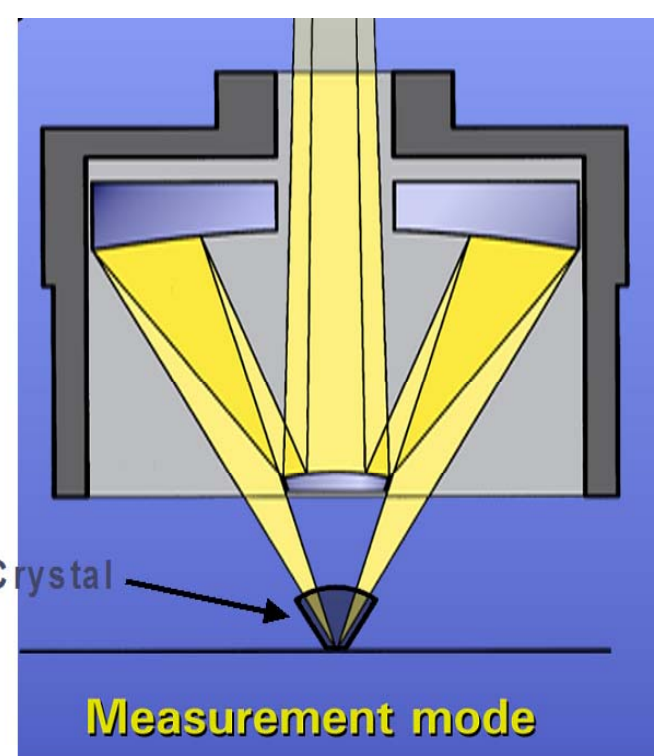

Measurement mode

Figure 1 - The left image shows the ATR objective in the viewing mode and the right image shows the ATR crystal lowered into the FOV for infrared analysis.

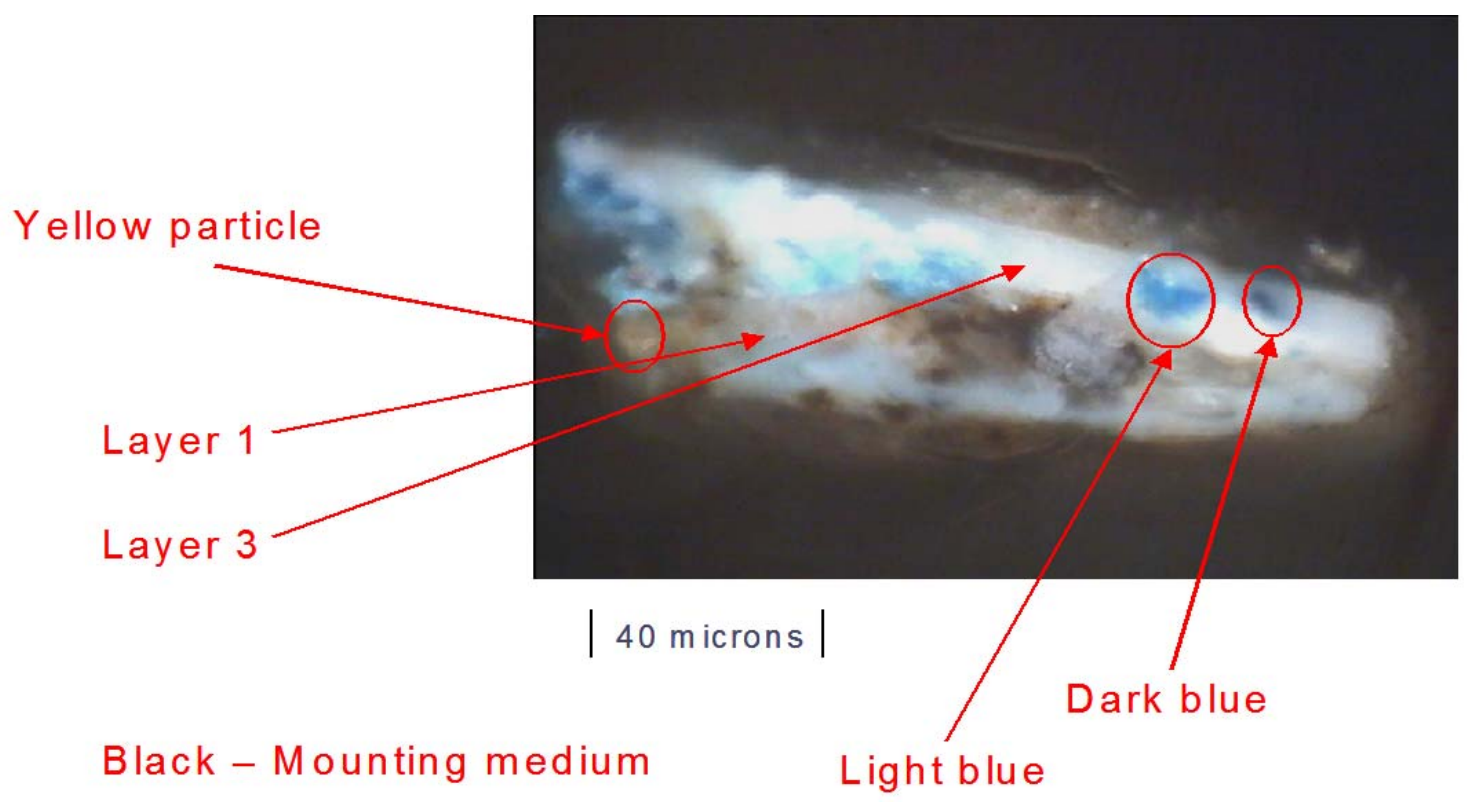

Figure 2 - Chip from an ancient work of art presented for ATR infrared microanalysis. 